\title{
Pesquisa Regulatória em Telecomunicações
}

Regulatory Research on Telecommunications

\section{A separação de poderes e o processo de institucionalização das agências reguladoras de telecomunicações nos EUA e no Brasil}

\author{
Coordenadas \\ Dissertação de Mestrado em Direito e Estado \\ Faculdade de Direito da Universidade de Brasília \\ Abril/2009, Brasília \\ Disponível em meio eletrônico: Biblioteca Digital \\ de Teses e Dissertações da UnB \\ (http://bdtd.bce.unb.br) \\ Autoria \\ Gabriel Boavista Laender \\ Mestre em Direito, Estado e Constituição pela UnB \\ Assessor do Gabinete Civil da Presidência da República e \\ Coordenador de Regulação do Grupo Téenico para \\ proposta do Plano Nacional de Banda Larga \\ Doutorando em Direito, Estado e Constituição (UnB)
}

\section{Resumo}

A presente dissertação analisa de que modo a separação de poderes e o modelo de freios e contrapesos influenciaram o processo de institucionalização da regulação de telecomunicações nos EUA e no Brasil, culminando com a criação da americana Federal Communications Commission (FCC) e da brasileira Agência Nacional de Telecomunicações (Anatel). Uma vez que a experiência do setor ferroviário foi determinante para a formatação institucional da regulação sobre a telefonia, o trabalho inicia pela avaliação daquele setor, onde descreve o processo que levou à institucionalização da Interstate Commerce Commission (ICC). Em seguida, o trabalho descreve o processo de institucionalização da FCC, bem como os eventos que levaram à reconfiguração dessa agência pelo Telecommunications Act de 1996. O estudo então se volta à experiência brasileira de telecomunicações, descrevendo o longo caminho de centralização operacional e normativa pelo governo federal, culminando com o monopólio das telecomunicações firmado pela Constituição de 1988. Finalmente, o estudo descreve o processo de ruptura institucional que resultou na criação da Anatel em 1997, e avalia a consolidação de suas características até o presente. A descrição busca enfatizar os problemas regulatórios trazidos especialmente no setor de telefonia e confrontá-los com a busca de soluções institucionais que propiciassem a implementação de políticas públicas de telecomunicação nos países paradigmas. Ao avaliar a busca de soluções institucionais, as relações entre os três poderes e entre os entes federados são identificadas. Ao final, com base na experiência relatada no setor de telecomunicações, o trabalho conclui sobre as implicações entre as características de institucionalização das atuais agências reguladoras e o 
princípio da separação de poderes, ponderando o processo de conformação dessas características frente à função de garantia institucional da liberdade exercida por aquele princípio.

\section{Abstract}

This dissertation analyses the way in which separation of powers and checks and balances have influenced the process of institutionalization of telecommunication regulation in the USA and in Brazil, leading to the creation of the American Federal Communications Commission (FCC) and the Brazilian Agência Nacional de Telecomunicações (Anatel). As the experience of the railway sector was paramount to the institutional framework of telephony regulation, the paper begins by evaluating that sector and describing the process which lead to the institutionalization of the Interstate Commerce Commission (ICC). It is followed by a description of the process of institutionalization of the FCC, as well as the events leading to the reframing of that agency by the Telecommunications Act of 1996. Then, the Brazilian experience is focused by the description of the long road of operational and normative centralization by the Federal Government leading to the monopoly of telecommunications established by the Constitution of 1988. Finally, the essay describes the process which resulted in the creation of Anatel in 1997 and evaluates the consolidation of its characteristics up to nowadays. The description purports to emphasize the regulatory problems brought especially by the sector of telephony and to confront them with the search of institutional solutions that could uphold the implementation of telecommunication public policies in the paradigm countries. When evaluating the search of institutional solutions, the relations among the three powers and among federal entities are identified. At last, based on the aforementioned experience of the telecommunication sector, the paper reaches a conclusion about the implications between the characteristics of the institutionalization of the present regulatory agencies and the principle of separation of powers, referring to the process of conformation of those characteristics vis-à-vis the role as institutional guarantee played by that principle. 


\section{A (in)adequação dos mecanismos regulatórios setoriais aos institutos jurídicos de índole constitucional do mercado e da universalização de serviços públicos}

\author{
Coordenadas \\ Dissertação de Mestrado em Direito e Estado \\ Faculdade de Direito da Universidade de Brasília \\ Agosto/2009, Brasília \\ Disponível em meio eletrônico: Biblioteca Digital de \\ Teses e Dissertações da UnB (http://bdtd.bce.unb.br)
}

\author{
Autoria \\ Renato Soares Peres Ferreira \\ Mestre em Direito, Estado e Constituição pela \\ Universidade de Brasília \\ Advogado
}

\section{Resumo}

A presente dissertação investiga de que maneira a regulação setorial instituída no Brasil compatibilizou-se ou deixou de se compatibilizar com os institutos jurídicos de índole constitucional do mercado e da universalização de serviços públicos. Adotou, para isso, como estudo de caso, a regulação do Serviço Telefônico Fixo Comutado (STFC) estabelecida, no setor de telecomunicações, a partir da privatização do sistema TELEBRÁS, analisando as principais linhas da política regulatória desse serviço até o ano de 2007. O trabalho cuida, assim, em um primeiro passo, da abordagem teórica da regulação, do mercado e da universalização, enquanto conceitos expressos jurídica e constitucionalmente no Brasil e, em seguida, analisa a configuração da regulação brasileira, diante das teorias e rationales regulatórias e do estudo de caso escolhido. Ao final, conclui-se pela adequação, ao menos relativa, entre as políticas regulatórias desenvolvidas para o STFC e os pressupostos constitucionais do mercado e da universalização, principalmente em razão da estratégia escolhida, de ampliar aos poucos a competição e exigir das empresas incumbentes o cumprimento, antes da ampliação definitiva da competição, de metas de universalização.

\section{Abstract}

This dissertation focuses on the Brazilian regulatory framework and its correlation with the constitutional principles of market and universal service. With this objective in mind, it zeroed in on the case of regulating "Fixed Switched Telephone Service" (STFC) after the privatization of the TELEBRÁS system, focusing on the main regulatory policies applied to this service until 2007. This research includes, as a first step, a theoretical study of regulation, market and universal service as concepts adopted by the Constitution of Brazil and, as a second step, analyses the Brazilian regulatory framework, according to theories of regulation and the selected case. As conclusion, this study states that there is a partial consistence between regulatory policies developed for the STFC and the constitutional 
values of market and universal service, especially due to the chosen strategy of light pro-competition regulation in the first moment and universal service obligations, in charge of the incumbent companies 
Pesquisas Acadêmicas no período de 2007 a 2009 em nível de pósgraduação lato sensu e stricto sensu

1. Alcance da não incidência da contribuição ao FUST, conforme prevista no parágrafo único do art. $6^{\circ}$ da Lei 9.998, de 2000/Sara de Sousa Coutinho/VI CURSO DE ESPECIALIZAÇÃ̃o EM REgULAÇÃo DE TELECOMUNICAÇÕES DA UNIVERSIDADE DE BRASílIA (2008).

2. Alterações do contrato de concessão do Serviço Telefônico Fixo Comutado, na modalidade local e a defesa do interesse do usuário/consumidor, As/Regina Lúcia Alves/VI CURSO DE ESPECIALIZAÇÃo EM RegULAÇÃo de TeLECOMUNICAÇões dA UNIVERSIDADE dE BRASílIA (2008).

3. Análise do cenário de competição no mercado brasileiro de capacidade espacial/Afonso Rocha Ferreira Junior/VI CURSO DE ESPECIALIZAÇÃO EM REGULAÇÃO DE TELECOMUNICAÇÕES DA UNIVERSIDADE DE BRASÍLIA (2008).

4. Análise microeconômica do estímulo ao crescimento do mercado de televisão por assinatura, utilizando dados do SATVA, do IPEADATA/Maria Augusta Bretas Lima/VI CURSO DE ESPECIALIZAÇÃo EM REGULAÇÃo de TELECOMUNICAÇÕES DA UnIVERSIDADE de BRASílIa (2008).

5. Aplicação da Metodologia de Gestão de Projetos no planejamento, execução, controle, relato e conclusão de missões de fiscalização na Agência Nacional de Telecomunicações - ANATEL/José Umberto Sverzut/VI CURSO de EsPECIALIZAÇÃo EM REGULAÇÃo de TeleCOMUNICAÇÕES DA UNIVERSIDADE DE BRASÍLIA (2008).

6. Aspectos Regulatórios, Mercadológicos e Tecnológicos da Digitalização da Radiodifusão Sonora na faixa de $\mathbf{3 0 - 3 0 0 0 ~} \mathrm{MHz}$ no Brasil/Elmano Rodrigues Pinheiro Filho/VI CURSO DE ESPECIALIZAÇÃO EM RegULAÇão de TELECOMUNICAÇõES dA UNIVERSIDADE DE BRASílIa (2008).

7. Atendimento aos usuários como reflexão para um novo marco regulatório nas telecomunicações brasileiras, $\mathrm{O} /$ Carlos Vinicius Brito Reis/VI CURSO de ESPECIALIZAÇÃo EM REgulaçÃo de TeleComunicações dA UNIVERSIDADE DE BRASÍLIA (2008).

8. Avaliação de abuso e eficiência no processo fiscalizatório da ANATEL/Wilson Bolcchi Junior/VI CURSO DE ESPECIALIZAÇÃO EM REGULAÇÃO dE TELECOMUNICAÇÕES DA UNIVERSIDADE DE BRASílIA (2008). 
9. Avaliação do modelo implantado a partir de 1998 tendo como referência a universalização do STFC/Marcelo Miranda Sampaio Corrêa/VI CURSO de ESPECIALIZAÇÃo EM REGULAÇÃO dE TELECOMUNICAÇÕES DA UNIVERSIDADE DE BRASÍLIA (2008).

10. Competição no mercado de serviço de telefonia fixa comutado na modalidade longa distância nacional (STFC-LDN) no Brasil, no período de 2000 a 2001: o problema da tarifa de utilização de rede local/Marco Antonio de Castro/VI CURSO de ESPECIALIZAÇÃo EM REGULAÇão DE TELECOMUNICAÇÕES DA UNIVERSIDADE DE BRASílIa (2008).

11. Competição nos Serviços Móveis: Revenda como uma alternativa/Maria Lucia Valadares e Silva/VI CURSO DE ESPECIALIZAÇÃO EM REGULAÇÃo de TELECOMUNICAÇÕES dA UNIVERSIDADE de BRASília (2008).

12. Congresso Nacional frente ao poder normativo das agências reguladoras: o caso da ANATEL, O/Renato Lima de Oliveira/VI CURSO DE ESPECIALIZAÇÃO EM REgULAÇÃO DE TELECOMUNICAÇÕES DA UNIVERSIDADE DE BRASÍLIA (2008).

13. Convergência de outorgas para exploração de serviços e redes de telecomunicações no Brasil/José de Sousa Paz Filho/VI CURSO DE ESPECIALIZAÇÃo EM REgULAÇÃo DE TELECOMUNICAÇõES DA UNIVERSIDADE DE BRASÍLIA (2008).

14. Custo de Capital Próprio no âmbito do novo contexto tarifário da telefonia fixa no Brasil: uma proposta de CAPM Global Modificado, O/Tulio Carné Bertini/VI CURSO dE EsPECIALIZAÇão EM REgULAÇÃo DE TELECOMUNICAÇÕES DA UNIVERSIDADE de BRAsílIa (2008).

15. Desafios regulatórios no setor de telecomunicação: as causas e conseqüências do controle político e da intervenção governamental na Agência Nacional de Telecomunicações/André Luiz Dias de Oliveira/VI CURSO de ESPECIALIZAÇÃo EM REGULAÇÃo de TeleCOMUNICAÇÕES DA UNIVERSIDADE DE BRASÍLIA (2008).

16. Diferenciação de regimes tributários como instrumento de assimetria regulatória no Serviço Telefônico Fixo Comutado - uma reflexão à luz dos precedentes do Supremo Tribunal Federal/Renato Soares Peres Ferreira/VI CURSO dE ESPECIALIZAÇÃo EM REGULAÇÃO dE TELECOMUNICAÇÕES DA UNIVERSIDADE DE BRASÍLIA (2008).

17. Direito à privacidade e a internet, $\mathrm{O} /$ Daniela Figueiredo Oliveira França/VI CURSO dE ESPECIALIZAÇÃo EM REGULAÇÃo dE TELECOMUNICAÇÕES DA UNIVERSIDADE DE BRASÍLIA (2008). 
18. Direitos e Garantias dos Usuários em Redes VolP/Davison Gonzaga da

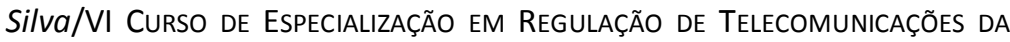
UNIVERSIDADE DE BRASílIa (2008).

19. Efeitos da não-cumulatividade do ICMS na determinação das tarifasteto da concessionária da Região III do Serviço Telefônico Fixo Comutado/João Batista Rodrigues Fonseca/VI CURSO DE ESPECIALIZAÇÃo EM REGULAÇÃO DE TELECOMUNICAÇÕES DA UNIVERSIDADE DE BRASÍLIA (2008).

20. Estudo de modelos alternativos ao CAPM para aplicação no WACC do setor de telecomunicações do Brasil/Caimi Franco Reis/VI CURSO DE ESPECIALIZAÇÃO EM REgULAÇÃO DE TELECOMUNICAÇÕES DA UNIVERSIDADE DE BRASÍlIA (2008).

21. Fraude em Telecomunicações: A responsabilidade das prestadoras de telecomunicações no combate a fraude/Luiz Roberto de Azevedo Moncorvo/VI CURSO de ESPECIALIZAÇÃo EM REGULAÇÃo dE TELECOMUNICAÇõES DA UNIVERSIDADE DE BRASÍLIA (2008).

22. Fraude no mercado de Telecomunicações Brasileiro/Luis Renato Giffoni Rodrigues/VI CURSO DE ESPECIALIZAÇÃO EM REGULAÇÃO DE TELECOMUNICAÇõES DA UNIVERSIDADE DE BRASílIA (2008).

23. Implantação do Sistema de Gerenciamento Centralizado: impactos sobre as obrigações das operadoras de serviços de telecomunicações nas interceptações telefônicas, A/Patrick Leonardo de Faria e Silva/VI

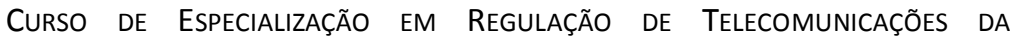
UNIVERSIDADE DE BRASÍLIA (2008).

24. Ingresso das Concessionárias do STFC no Mercado de TV por Assinatura/Fabrício Guimarães Madruga Lopes/VI CURSO DE ESPECIALIZAÇÃo EM REgULAÇÃo dE TELECOMUNICAÇÕES dA UNIVERSIDADE DE BRASÍLIA (2008).

25. Long Run Incremental Cost (LRIC) aplicado ao cálculo das tarifas de interconexão nas telecomunicações: desafios e resultados na implantação do modelo no Brasil/Jorge Ernesto Sanchez Ruiz/VI CURSO DE EsPECIALIZAÇão EM REGULAÇ̃̃o DE TELECOMUNICAÇõeS DA UNIVERSIDADE DE BRASÍLIA (2008).

26. Mercado de certificação compulsória de produtos de telecomunicações: políticas, grupos de interesse e regulação/Vladimir Daigele Simões Barbosa/VI CURSO DE ESPECIALIZAÇÃo EM REGULAÇÃO DE TeleComunicações da Universidade de Brasília (2008).

27. Mobilidade restrita: regra de transição para a convergência dos serviços fixos e móveis/Renata Figueiredo Santoyo/VI CURSO DE 
Especialização em Regulação de Telecomunicações da Universidade de BRASÍLIA (2008).

28. Modelo de aplicação do Fundo de Universalização dos Serviços de Telecomunicações (FUST): o caso do PMU-I, Um/Enilce Nara Versiani/VI CURSO de ESPECIALIZAÇÃo EM REGULAÇÃo dE TELECOMUNICAÇÕES DA UNIVERSIDADE DE BRASílIA (2008).

29. Modelo sustentável para implantação de redes municipais sem fio de acesso à internet, Um/Daniel Brandão Cavalcanti/VI CURSO DE ESPECIALIZAÇÃO EM REGULAÇÃo DE TELECOMUNICAÇÕES DA UNIVERSIDADE DE BRASÍlIA (2008).

30. Preços de acesso e regulação tarifária baseada em custos/Daniel

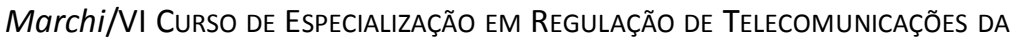
UNIVERSIDADE DE BRASÍLIA (2008).

31. Princípio da insignificância e as rádios comunitárias clandestinas: uma visão jurisprudencial, O/Gesiléa Fonseca Teles/VI CURSO DE ESPECIALIZAÇÃO EM REGULAÇÃo dE TELECOMUNICAÇ̃̃ES DA UNIVERSIDADE DE BRASílIa (2008).

32. Proposta metodológica de Plano de Negócio para leilões de espectro de radiofrequência no Brasil, Uma/Pedro Borges Griese/VI CURSO DE ESPECIALIZAÇÃo EM REGULAÇÃo DE TELECOMUNICAÇÕES DA UNIVERSIDADE DE BRASÍLIA (2008).

33. Reforma da Lei Geral de Telecomunicações: tópicos fundamentais/Marcel Netto de Lima/VI CURSO DE ESPECIALIZAÇÃO EM REGULAÇÃo de TELECOMUNICAÇÕES DA UNIVERSIDADE de BRASílIa (2008).

34. Regulação e Qualidade do Serviço de Telefonia Fixa no Brasil/Marco Antonio Santana Gomes/VI CURSO DE ESPECIALIZAÇÃO EM REGULAÇÃO DE TeleCOMUNICAÇÕES DA UNIVERSIDADE de BRAsílIa (2008).

35. Regulação Setorial e Convergência Tecnológica: Um estudo sobre o modelo regulatório do Reino Unido/Bernardo Fernandes Correa Mendonça/VI CURSO de ESPECIALIZAÇÃo EM REgULAÇÃo de TELECOMUNICAÇõES DA UNIVERSIDADE DE BRASílIA (2008).

36. Serviço Móvel Pessoal e Serviço de Comunicação Multimídia: fusão em novo serviço/Nilo Pasquali/VI CURSO dE ESPECIALIZAÇÃo EM REGULAÇão DE TELECOMUNICAÇÕES DA UNIVERSIDADE DE BRASílIA (2008).

37. Spectrum trading e gerência do espectro: a experiência internacional e o caso brasileiro/Raphael Garcia de Souza/VI CURSO DE ESPECIALIZAÇÃo

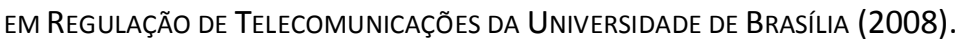


38. Subconcessão e a revenda no contrato de concessão da ANATEL/Andreza Nascimento de Lemos/VI CURSO DE ESPECIALIZAÇÃo EM

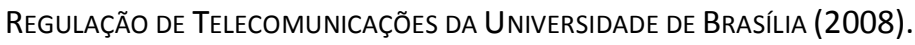

39. Tecnologia WIMAX e regulação do acesso à internet banda larga: o caso brasileiro/Gustavo Tavares Grillo/VI CURSO DE ESPECIALIZAÇÃO EM REGULAÇÃO DE TELECOMUNICAÇÕES DA UNIVERSIDADE DE BRASílIA (2008).

40. TV Pública no Brasil: a criação da TV Brasil e sua inserção no modo de regulação setorial da televisão brasileira/Jonas Chagas Lúcio

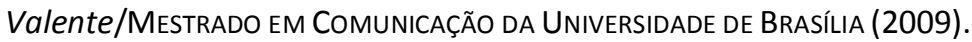

41. Regime público em discussão: como as alterações quinquenais do contrato de concessão podem auxiliar o regulador na evolução da prestação do serviço de telecomunicações no Brasil, O/Secundino da Costa Lemos/VI CURSO dE ESPECIALIZAÇÃo EM REgULAÇÃo DE TELECOMUNICAÇÕES DA UNIVERSIDADE DE BRASílIA (2008).

42. Soluções de curto e médio prazo para evitar a prescrição intercorrente em processos administrativos instaurados na Agência Nacional de Telecomunicações - ANATEL/Willy da Cruz Moura/VI CuRso de EspeCializaçÃo em RegulaçÃo de Telecomunicações dA UNIVERSIDADE DE BRASÍLIA (2008).

43. Prescrição intercorrente no Procedimento para Apuração de Descumprimento de Obrigação (PADO) na ANATEL/Janaína Diniz da Gama/VI CURSO dE ESPECIALIZAÇÃo EM REGULAÇão dE TELECOMUNICAÇÕES DA UNIVERSIDADE DE BRASílIA (2008).

44. Universalização dos serviços de telecomunicações/Moisés Gonçalves/VI CURSO DE ESPECIALIZAÇÃO EM REGULAÇÃo DE TELECOMUNICAÇõES DA UNIVERSIDADE DE BRASÍLIA (2008). 
Trabalhos de Conclusão de Curso de Graduação em Direito e de Iniciação Científica

1. Fundamentalidade do direito à comunicação: internet e participação no contexto da sociedade em rede. Políticas públicas de acesso à internet no Brasil, A/Pedro Augusto Maia Felizola/TrABaLHO DE Conclusão de Curso de Graduação em Direito da Universiddade de BrasílIA (2009).

2. Radiodifusão como serviço público: consequências da definição da readiodifusão como serviço público na possibilidade de imposição de cotas de conteúdo nacional, A/Laura Fernandes de Lima Lira/TRABALHO de Conclusão de Curso de Graduação em DiReito da Universidade de BRASÍlia (2009).

3. Regulação jurídica e empreendedorismo: impactos jurídicos do conceito de empreendedorismo no grau de concentração regulatória normativa no setor de telecomunicações mediante estudo do caso Actium/André Moura Gomes/TrabalHo de CONCLUSÃo DE CURSO DE GRAduação em Direito da UniversidAde de Brasília (2009).

4. Serviços titularizados pelo Estado e "regime privado"/Rodrigo de Oliveira Fernandes/Trabalho de Conclusão de CURSO de Graduação em DiREITO DA UNIVERSIDADE DE BRASílIA (2009). 